\title{
Methanol extracts of Euphorbia cooperi inhibit the production of inflammatory mediators by inhibiting the activation of c-Jun N-terminal kinase and p38 in murine macrophages
}

\author{
YOUNG-CHANG CHO, IN-SEON LEE, HUIYUN SEO, ANNA JU, DEOKKYU YOUN, \\ YOUNGHYUN KIM, JAEHEE CHOUN and SAYEON CHO \\ College of Pharmacy, Chung-Ang University, Seoul 156-756, Republic of Korea
}

Received March 23, 2014; Accepted July 22, 2014

DOI: $10.3892 / \mathrm{mmr} .2014 .2560$

\begin{abstract}
Numerous Euphorbiaceae plants have been used for the treatment of diseases, including liver diseases, asthma and rheumatism. The present study evaluated the effect of methanol extracts from Euphorbia cooperi (MEC), a member of the Euphorbiaceae plant family, on the production of inflammatory cytokines interleukin (IL)-6 and tumor necrosis factor (TNF)- $\alpha$, nitric oxide (NO) as well as the activation of mitogen-activated protein kinase and nuclear factor $(\mathrm{NF})-\kappa \mathrm{B}$ signaling. Non-cytotoxic concentrations of MEC significantly reduced the production of NO and IL-6, but not TNF- $\alpha$, in lipopolysaccharide (LPS)-stimulated RAW 264.7 macrophages. The decreased production of NO by MEC was due to alleviated expression of inducible NO synthase. Reporter assays with cells treated with MEC demonstrated reduced activator protein-1 (AP-1) activity, while NF- $\kappa$ B activity was not reduced. Furthermore, the phosphorylation levels of c-Jun N-terminal kinase (JNK) and p38 were suppressed by MEC while phosphorylation levels of inhibitor of $\kappa \mathrm{B}$ were not reduced by $\mathrm{MEC}$, suggesting that $\mathrm{MEC}$-mediated inactivation of JNK and p38 is the underlying regulatory mechanism for inflammatory mediators in LPS-stimulated RAW 264.7 macrophages.
\end{abstract}

\section{Introduction}

Macrophages are the primary effector cells of the immune system that protect against microbial infection. Upon stimulation with pathogen-derived molecules, including

Correspondence to: Professor Sayeon Cho, College of Pharmacy, Chung-Ang University, 84 Heukseok-Ro, Seoul 156-756, Republic of Korea

E-mail: sycho@cau.ac.kr

Key words: methanol extracts of Euphorbia cooperi, nitric oxide, inducible nitric oxide synthase, interleukin 6, tumor necrosis factor- $\alpha$, p38 mitogen-activated protein kinase, c-Jun $\mathrm{N}$-terminal kinase lipopolysaccharide (LPS) and flagellin, macrophages secrete a variety of inflammatory mediators and cytokines $(1,2)$. These secreted proteins bind tightly to toll-like receptors (TLRs) and stimulate the formation of signaling complexes resulting in a rapid defensive response (3-6). The tight regulation of host immune signaling ensures that the resulting immune response is appropriate for the continuously changing microenvironment, as well as for maintenance of immunological balance. However, inappropriate or prolonged activation of the immune system is largely responsible for the pathology of acute and chronic inflammatory conditions, including septic shock and chronic inflammatory conditions, such as rheumatoid arthritis, inflammatory bowel disease and chronic obstructive pulmonary disease $(1,7)$. In this regard, the secretion of pro-inflammatory mediators by activated macrophages, including cytokines, growth factors, hydrolytic enzymes, bioactive lipids, reactive oxygen intermediates and nitric oxide (NO), are involved in the pathogenesis of tissue injury $(1,3)$. Therefore, identification of agents that can regulate the production of pro-inflammatory mediators is considered an effective strategy for developing therapeutic agents to treat severe inflammation.

Activated macrophages transcriptionally express inducible NO synthase (iNOS) in response to various pro-inflammatory cytokines and bacterial LPS, resulting in the production of NO via the oxidative deamination of L-arginine at sites of inflammation $(8,9)$. NO modulates a variety of biological processes, including inflammation and carcinogenesis $(10,11)$. Tumor necrosis factor- $\alpha$ (TNF- $\alpha$ ) and interleukin (IL)- 6 are potent pro-inflammatory cytokines that are important for stimulating the secretion of additional inflammatory cytokines. Therefore, inhibiting the excessive production of these mediators in macrophages through the inhibition of mRNA and protein expression of iNOS, TNF- $\alpha$ and IL- 6 may be a viable strategy for the development of novel anti-inflammatory agents.

Several attempts have been made to develop a new generation of anti-inflammatory agents from natural compounds, as natural compounds are known to have fewer side effects (12). The latexes of Euphorbia plants were traditionally considered as toxic substances due to their irritancy on mucus membranes, including the nose and mouth, in humans (13). Although Euphorbia plants have toxic effects in humans, the extracts of Euphorbia plants are well known to inhibit excessive 
inflammation and are used in Chinese medicine $(14,15)$. Suarez et al demonstrated that intraperitoneal administration of an aqueous extract of Croton malambo (Euphorbiaceae) resulted in a significant anti-inflammatory effect in a rat model of edema (16). Furthermore, Sangre de Drago (dragon's blood) from Croton lechleri (Euphorbiaceae) inhibits inflammation in vitro and in vivo $(17,18)$. For example, treatment with Sangre de Drago significantly decreased intracellular generation of reactive oxygen species in several cell lines and alleviated paw edema in rats (18). However, the anti-inflammatory effects of methanol extracts of Euphorbia cooperi (MEC) remain to be elucidated.

In the present study, the anti-inflammatory effect of MEC in LPS-stimulated RAW 264.7 macrophages and its underlying mechanisms were investigated to evaluate the therapeutic potential of MEC in the treatment of abnormal inflammation.

\section{Materials and methods}

Cell culture and reagents. The RAW 264.7 macrophages, a mouse monocytic cell line, were cultured in Dulbecco's modified Eagle's medium supplemented with $10 \%$ fetal bovine serum, $50 \mathrm{U} / \mathrm{ml}$ penicillin and $50 \mu \mathrm{g} / \mathrm{ml}$ streptomycin (Gibco-BRL, Grand Island, NY, USA) at $37^{\circ} \mathrm{C}$ in a $5 \% \mathrm{CO}_{2}$ humidified air atmosphere. Rabbit polyclonal anti-inhibitors of $\kappa \mathrm{B}(\mathrm{I} \kappa \mathrm{B})$ and mouse anti-tubulin antibodies were purchased from Santa Cruz Biotechnology, Inc. (Santa Cruz, CA, USA). Rabbit polyclonal anti-inducible iNOS, rabbit polyclonal anti-phospho IкB, rabbit polyclonal anti-phospho p38 mitogen-activated protein kinase (MAPK), rabbit polyclonal anti-p38, mouse monoclonal anti-phospho extracellular signal-regulated kinase (ERK), rabbit polyclonal anti-ERK, rabbit polyclonal anti-phospho c-Jun $\mathrm{N}$-terminal kinase (JNK) and mouse monoclonal anti-JNK were purchased from Cell Signaling Technology Inc. (Danvers, MA, USA). MEC was purchased from the International Biological Material Research Center (Daejeon, Korea). The above compounds were dissolved in dimethyl sulfoxide (DMSO; Sigma-Aldrich, St. Louis, MO, USA) and added directly to the culture media. The final concentrations of DMSO never exceeded $0.1 \%$, which did not affect the assay systems.

3-(4,5-dimethylthiazol-2-yl)-2,5-diphenyltetrazolium bromide (MTT) assay. The RAW 264.7 macrophages were incubated with MEC and LPS for $24 \mathrm{~h}$. Following incubation, MTT $(0.5 \mathrm{mg} / \mathrm{ml})$ was added for $3 \mathrm{~h}$ at $37^{\circ} \mathrm{C}$ and the supernatants were carefully removed. The crystals of viable cells were dissolved in DMSO and absorbance was measured at $595 \mathrm{~nm}$ using a Synergy microplate reader (BioTek Instruments Inc., Winooski, VT, USA).

Nitrite assay. The RAW 264.7 macrophages were incubated with MEC and LPS for $24 \mathrm{~h}$. Following incubation, the levels of NO synthesis were determined by assaying the culture supernatants for nitrite, the stable reaction product of NO, with molecular oxygen, using the Griess reagent ( $1 \%$ sulfanilamide, $0.1 \% \mathrm{~N}-1$-naphthylenediamine dihydrochloride and $2.5 \%$ phosphoric acid). The absorbance was measured at $540 \mathrm{~nm}$ using a Synergy microplate reader after $10 \mathrm{~min}$ incubation.
Enzyme-linked immunosorbent assay (ELISA). The RAW 264.7 macrophages were stimulated with LPS and MEC for $24 \mathrm{~h}$. Following stimulation, the supernatants were obtained and the quantities of TNF- $\alpha$ and IL- 6 in the culture supernatants were determined using sandwich ELISA, which used monoclonal antibodies specific to each mediator. Prior to the application of samples, the plate was pre-coated with coating antibody in the supplied buffer. Following overnight incubation at $4^{\circ} \mathrm{C}$, the plate was washed and assay diluents $(1 \mathrm{X})$ were treated for $1 \mathrm{~h}$. The samples were then loaded into each well and incubated for $2 \mathrm{~h}$ at room temperature. They were then treated with biotinylated secondary antibody and horseradish peroxidase-streptavidin solutions for $1 \mathrm{~h}$ and $30 \mathrm{~min}$, respectively and substrate solution was added to the washed plate. After $10 \mathrm{~min}$ incubation in the dark, $1 \mathrm{~N} \mathrm{H}_{3} \mathrm{PO}_{4}$ treatment was applied and the optical density of the individual wells was determined at $450 \mathrm{~nm}$ using a Synergy microplate reader.

Reverse transcription polymerase chain reaction (RT-PCR). Total RNA was prepared from the cells and reverse-transcribed into complementary DNA (cDNA), following which PCR amplification of the cDNA was performed. The sequences of PCR primers used in the present study were as follows: mouse iNOS, forward 5'-GCA TGG AAC AGT ATA AGG CAA ACA-3' and reverse 5'-GTT TCT GGT CGA TGT CAT GAG CAA-3'; TNF- $\alpha$, forward 5'-GTG CCA GCC GAT GGG TTG TAC C-3' and reverse 5'-AGG CCC ACA GTC CAG GTC ACT G3'; IL-6, forward 5'-TCT TGG GAC TGA TGC TGG TGA C-3' and reverse 5'-CAT AAC GCA CTA GGT TTG CCG A-3' and GAPDH, forward 5'-GTC TTC ACC ACC ATG GAG AAG G-3' and reverse 5'-CCT GCT TCA CCA CCT TCT TGC C-3'. The PCR was run for $20-25$ cycles of $94^{\circ} \mathrm{C}(30 \mathrm{sec}), 60^{\circ} \mathrm{C}$ $(30 \mathrm{sec})$ and $72^{\circ} \mathrm{C}(30 \mathrm{sec})$ using a Bioer's thermal cycler (Bioer Technology Co., Hangzhou, China). Following amplification, the RT-PCR products $(10 \mu \mathrm{l})$ were separated in $1.5 \%(\mathrm{w} / \mathrm{v})$ agarose gels and stained with ethidium bromide.

Transient transfection and luciferase assay. The nuclear factor-kappa B (NF- $\kappa \mathrm{B})$ and activator protein-1 (AP-1) promoters containing the luciferase gene were purchased from Agilent Technologies (Santa Clara, CA, USA). HEK 293 cells were transiently transfected using polyethyleneimine according to the manufacturer's instructions (Polysciences Inc., Warrington, PA, USA) and transfected cells were stimulated with phorbol 12-myristate 13-acetate (PMA) in the presence or absence of MEC for $24 \mathrm{~h}$. The cells were harvested and the luciferase activities were assayed according to the manufacturer's instructions (Promega Corporation, Madison, WI, USA).

Preparation of total cell lysates. LPS-stimulated RAW 264.7 cells were treated with MEC for the indicated time periods (15 min and $24 \mathrm{~h}$ ) and washed with ice-cold phosphate-buffered saline. The cells were lysed in lysis buffer containing $0.5 \%$ NP-40, $0.5 \%$ Triton X-100, $150 \mathrm{mM}$ sodium chloride, $20 \mathrm{mM}$ trisaminomethane-hydrochloride (Tris- $\mathrm{HCl}$; $\mathrm{pH} 8.0), 1 \mathrm{mM}$ ethylenediaminetetraacetic acid, $1 \%$ glycerol, $1 \mathrm{mM}$ phenylmethylsulfonyl fluoride and $1 \mu \mathrm{g} / \mathrm{ml}$ aprotinin, collected into microtubes and then centrifuged at $15,500 \mathrm{x} \mathrm{g}$ for $30 \mathrm{~min}$ at $4^{\circ} \mathrm{C}$. The supernatants were prepared in new microtubes. 
Western blot analysis. Protein concentration was measured using the Bradford method. Aliquots of the cell lysates were separated on a $10 \%$ sodium dodecyl sulfate-polyacrylamide gel in a Mini-Protein II gel apparatus (Bio-Rad, Richmond, CA, USA) and transferred onto nitrocellulose membranes (GE Healthcare, Milwaukee, WI, USA) with transfer buffer [(192 mM glycine, $25 \mathrm{mM}$ Tris-HCl; pH 8.8 and $20 \% \mathrm{MeOH}$ (v/v)]. Following inhibition of the non-specific sites with $5 \%$ bovine serum albumin solution, the membrane was incubated overnight at $4^{\circ} \mathrm{C}$ with the primary antibodies $(1: 1,000)$. Each membrane was further incubated for $1 \mathrm{~h}$ with secondary peroxidase-conjugated goat polyclonal immunoglobulin G (IgG; 1:5,000). The target proteins were detected using an enhanced chemiluminescence solution.

Statistical analysis. Differences between the experimental conditions were assessed using Student's t-test. $\mathrm{P}<0.05$ was considered to indicate a statistically significant difference. In all instances, the means of data from three independent experiments were analyzed.

\section{Results}

Effects of MEC on the viability of activated macrophages. To determine the maximal effective concentration of MEC that has minimal cytotoxicity, RAW 264.7 macrophages were treated with the indicated concentrations of $\operatorname{MEC}(20,50,100$ and $200 \mu \mathrm{g} / \mathrm{ml}$ ) for $24 \mathrm{~h}$ in the presence of LPS. Cell viability was determined by the ability of the cells to metabolically reduce a tetrazolium salt to a formazan dye. MEC had little effect on cell viability at doses of $\leq 100 \mu \mathrm{g} / \mathrm{ml}$ either in the absence or presence of $0.5 \mu \mathrm{g} / \mathrm{ml}$ LPS (Fig. 1). However, significant cytotoxicity was observed at MEC concentrations $\geq 100 \mu \mathrm{g} / \mathrm{ml}$. These data indicated that low doses of MEC $(100 \mu \mathrm{g} / \mathrm{ml})$ do not affect the viability of RAW 264.7 macrophages. Therefore, concentrations $\leq 100 \mu \mathrm{g} / \mathrm{ml}$ were used in the subsequent experiments.

Effects of MEC on the production of LPS-induced iNOS and NO. To determine the anti-inflammatory effect of MEC, the release of NO was examined by treating the RAW 264.7 macrophages with MEC in the absence or presence of LPS. Culture supernatants were collected after $24 \mathrm{~h}$ incubation and the quantity of nitrite accumulated in the culture media was estimated using Griess reagent as an indicator of NO release. As shown in Fig. 2A, the nitrite concentration in the media was increased markedly in the LPS-activated RAW 264.7 macrophages compared with the unstimulated cells. When the RAW 264.7 macrophages were treated with various concentrations of MEC, the levels of LPS-stimulated nitrite production decreased significantly in a dose-dependent manner (Fig. 2A). Since nitrite is a product of iNOS activation, the effects of MEC on iNOS mRNA and protein in RAW 264.7 macrophages were measured using RT-PCR and western blot analysis, respectively. As shown in Fig. 2B, treatment of RAW 264.7 macrophages with various concentrations of MEC markedly reduced the LPS-stimulated increase in the level of $i N O S$ mRNA expression in a dose-dependent manner. Furthermore, MEC treatment reduced the LPS-induced increase of iNOS protein expression in these cells (Fig. 2C). These results indicated that MEC reduced NO production

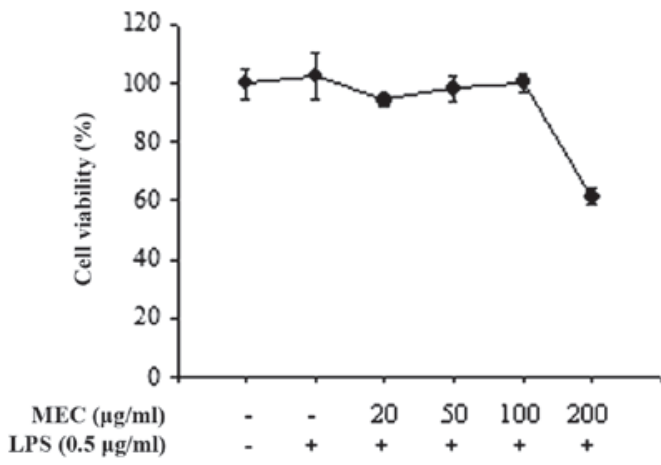

Figure 1. Effect of MEC on cell viability. RAW 264.7 macrophages were treated with LPS in the presence of $\operatorname{MEC}(20,50,100$ and $200 \mu \mathrm{g} / \mathrm{ml})$. After incubation for $24 \mathrm{~h}$, cell viability was measured using a 3-(4,5-dimethylthiazol-2-yl)-2,5-diphenyltetrazolium bromide assay. Cell viability of each group was compared with that of the LPS-treated group and presented as a graph. Data are expressed as the mean \pm standard deviation $(n=3)$. MEC, methanol extracts from Euphorbia cooperi; LPS, lipopolysaccharide.

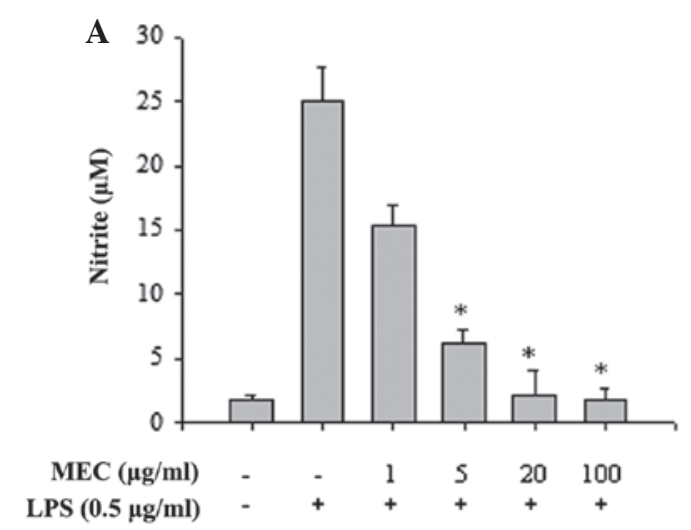

$\mathbf{B}$

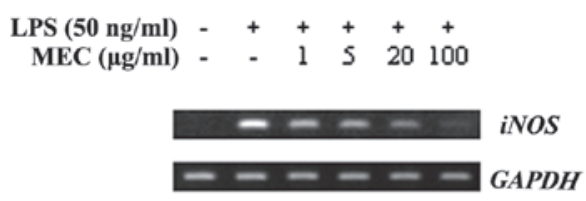

C

$\operatorname{LPS}(1 \mu \mathrm{g} / \mathrm{ml})-+++++$

$\operatorname{MEC}(\mu \mathrm{g} / \mathrm{ml})$ - $\quad-\quad 1 \quad 520100$

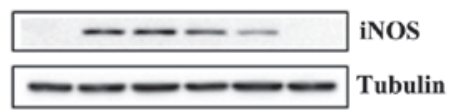

Figure 2. Inhibitory effect of MEC on the production of NO. RAW 264.7 macrophages were stimulated with LPS in the presence of MEC $(1,5,20$ and $100 \mu \mathrm{g} / \mathrm{ml}$ ) for the indicated time periods. (A) After $24 \mathrm{~h}$ stimulation, NO secretion in supernatants was measured using Griess reagent. NO secretion was calculated by a standard curve using nitrite standard solution. The data are expressed as the mean \pm standard deviation $(n=3) .{ }^{*} \mathrm{P}<0.05$, relative to an LPS-treated control group. (B) After $6 \mathrm{~h}$ stimulation, total RNA was extracted and reverse transcribed to cDNA. iNOS was amplified by polymerase chain reaction and detected using a gel documentary system. Expression of GAPDH was used as an internal control. (C) Total cell lysates were prepared after $24 \mathrm{~h}$ stimulation and western blot analysis was performed. The expression levels of iNOS protein were detected by enhanced chemiluminescence and normalized using tubulin as a loading control. MEC, methanol extracts from Euphorbia cooperi; NO, nitric oxide; LPS, lipopolysaccharide; iNOS, inducible NO synthase. 
A

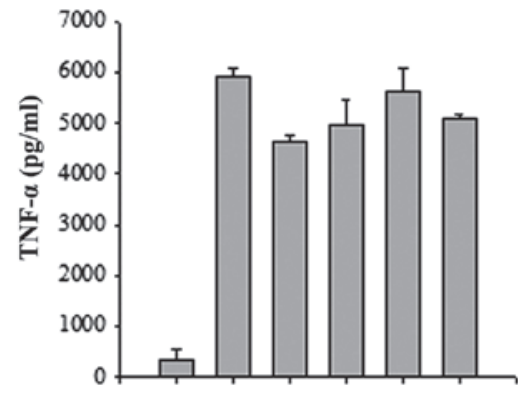

$\operatorname{MEC}(\mu \mathrm{g} / \mathrm{ml})$. LPS $(0.5 \mu \mathrm{g} / \mathrm{ml})$.

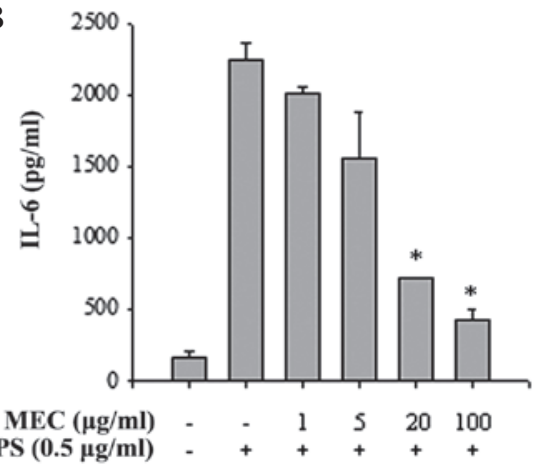

$\mathbf{C}$

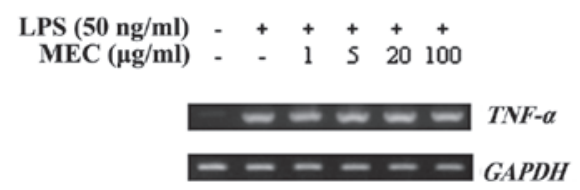

D

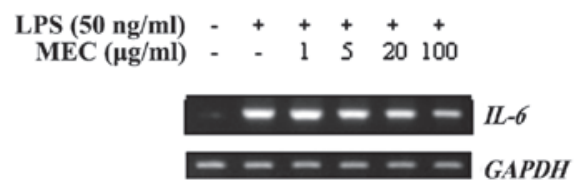

Figure 3. Inhibitory effect of MEC on the production of pro-inflammatory cytokines. RAW 264.7 macrophages were stimulated with LPS in the presence of MEC $(1,5,20$ and $100 \mu \mathrm{g} / \mathrm{ml})$ for the indicated time periods. After $24 \mathrm{~h}$ stimulation, levels of (A) TNF- $\alpha$ and (B) IL-6 in supernatants were measured by enzyme-linked immunosorbent assay. Secretion levels of each cytokine were determined using a standard curve and presented as a graph. The data are expressed as the mean \pm standard deviation $(\mathrm{n}=3)$. ${ }^{*} \mathrm{P}<0.05$, relative to the LPS-treated control group. After $6 \mathrm{~h}$ stimulation, total RNA was extracted and reverse transcribed to cDNA. (C) $T N F-\alpha$ and (D) $I L-6$ mRNA was amplified by polymerase chain reaction and detected using a gel documentary system. GAPDH expression was used as a loading control. MEC, methanol extracts from Euphorbia cooperi; LPS, lipopolysaccharide; TNF, tumor necrosis factor; IL, interleukin.

by inhibiting the expression of iNOS in LPS-activated macrophages.

Differential inhibitory effect of MEC on the production of $I L-6$ and TNF- $\alpha$ in activated macrophages. Since stimulation of macrophages with LPS consequently induces the production of pro-inflammatory cytokines, including TNF- $\alpha$ and IL-6, the anti-inflammatory effects of MEC on cytokine production were evaluated in LPS-stimulated macrophages. As shown in Fig. 3A and B, LPS-stimulated RAW 264.7 macrophages produced large quantities of TNF- $\alpha$ and IL-6. Notably, MEC treatment reduced the LPS-stimulated production of IL-6 in a dose-dependent manner but did not affect the production of TNF- $\alpha$. Furthermore, the LPS-induced increase in $I L-6$ mRNA expression was also markedly inhibited by MEC treatment, whereas the mRNA level of TNF- $\alpha$ was not affected (Fig. 3C and D). These results indicated that MEC selectively regulated the production of IL- 6 by inhibiting $I L-6$ mRNA expression.

Selective inhibition of MAPK phosphorylation by MEC in activated macrophages. To elucidate the mechanisms underlying the anti-inflammatory effect of MEC, the activities of the inflammation-associated transcription factors, NF- $\mathrm{\kappa B}$ and AP-1, were measured. HEK 293 cells transiently transfected with NF- $\kappa$ B or AP-1 luciferase reporter constructs were treated with PMA, either in the absence or presence of MEC and luciferase activity was determined. MEC had no apparent effect on PMA-stimulated NF-kB activity (Fig. 4A), however, the constructs were markedly stimulated by PMA and the PMA-activated AP-1 transcriptional activity was inhibited by MEC in a dose-dependent manner (Fig. 4B). To investigate this further, the effects of MEC on the LPS-induced
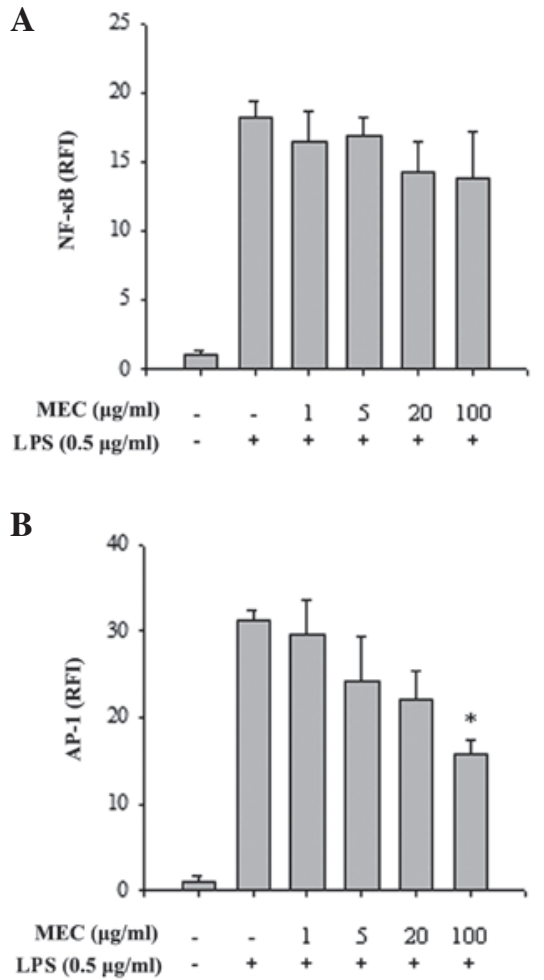

Figure 4. Inhibitory effect of MEC on NF- $\mathrm{KB}$ and $\mathrm{AP}-1$ promoter activity. HEK 293 cells were transiently transfected with (A) NF-kB and (B) AP-1 with $\beta$-galactosidase as an internal control. After $24 \mathrm{~h}$ incubation, cells were stimulated with LPS in the presence of MEC $(1,5,20$ and $100 \mu \mathrm{g} / \mathrm{ml})$ for $24 \mathrm{~h}$. Cell lysates were prepared using passive lysis buffer and luminescence values were measured using a microplate reader. RFI compared with the unstimulated group is presented as a graph. The data are expressed as the mean \pm standard deviation $(\mathrm{n}=3)$. ${ }^{*} \mathrm{P}<0.05$, relative to the LPS-treated control group. MEC, methanol extracts from Euphorbia cooperi; NF- $\mathrm{kB}$, nuclear factor- $\mathrm{kB}$; AP-1, activator protein 1; LPS, lipopolysaccharide; RFI, relative fold induction. 
A

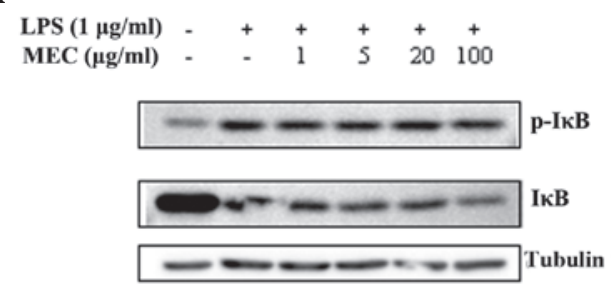

B

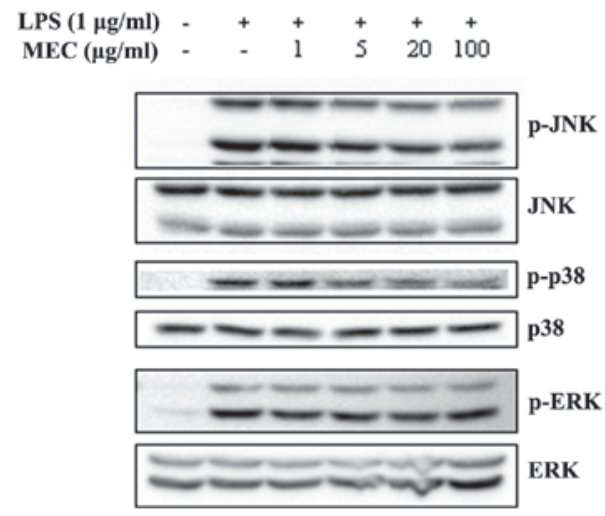

Figure 5. Inhibitory effect of MEC on the activation of NF- $\kappa \mathrm{B}$ and MAPKs. RAW 264.7 macrophages were pretreated with various concentrations of $\operatorname{MEC}(1,5,20$ and $100 \mu \mathrm{g} / \mathrm{ml})$ for $1 \mathrm{~h}$ and then stimulated with LPS for $15 \mathrm{~min}$. Total cell lysates were prepared and western blot analysis was performed.

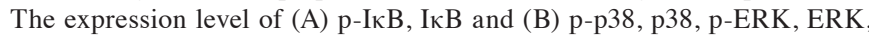
p-JNK and JNK was detected by each specific antibody and tubulin was used as a loading control. MEC, methanol extracts from Euphorbia cooperi; $\mathrm{NF}-\kappa \mathrm{B}$, nuclear factor- $\kappa \mathrm{B}$; MAPK, mitogen-activated protein kinase; LPS, lipopolysaccharide; p-, phosphorylated; ERK, extracellular signal-regulated kinase; JNK, c-Jun N-terminal kinase; I $\kappa$ B, inhibitor of $\kappa \mathrm{B}$.

phosphorylation of I $\mathrm{B}$ and MAPKs in RAW 264.7 cells were analyzed using western blot analysis. LPS treatment markedly induced the phosphorylation of I $\mathrm{B}$ and MAPKs, whereas MEC inhibited the increased phosphorylation of JNK and p38 in a dose-dependent manner. By contrast, the LPS-stimulated phosphorylation of I $\kappa$ B and ERK was not affected by MEC treatment (Fig. 5). The results indicated that inflammatory responses activated through MAPK signaling pathways, particularly those involving JNK and p38, are sensitive to inhibition by MEC.

\section{Discussion}

The excessive production of iNOS by external stimuli is recognized as an important pathophysiological consequence in numerous inflammatory disorders (11). Aberrant expression of iNOS leads to abnormal levels of NO production $(8,19,20)$ and several studies have indicated that increased expression of iNOS is associated with carcinogenesis and severe inflammatory diseases, including sepsis and arthritis (10,21,22). Since iNOS/NO are central to the inflammatory process, a number of studies have attempted to identify novel anti-inflammatory agents that inhibit the expression of iNOS and to elucidate the underlying mechanism. The present study demonstrated that MEC inhibited the production of NO in RAW 264.7 macrophages by inhibiting the expression of iNOS in a non-cytotoxic manner. These results suggested that MEC contains anti-inflammatory phytochemicals.
Pro-inflammatory cytokines are key mediators of apoptosis and innate immune reactions. Excessive levels of pro-inflammatory cytokines can induce tissue injury and potentiate septic shock $(23,24)$. Therefore, agents that inhibit the production and action of pro-inflammatory cytokines may inhibit the progression of inflammatory diseases. The pro-inflammatory cytokines, TNF- $\alpha$ and IL-6, are major pathogenic factors for a number of inflammatory diseases, including rheumatoid arthritis, and anti-IL-6 receptor antibody is currently used as a therapeutic agent in the clinical treatment of this disease (24-26). The present study demonstrated that MEC significantly inhibited the production of IL- 6 , but not TNF- $\alpha$, in LPS-stimulated RAW 264.7 macrophages. Several studies have demonstrated that these cytokines are not simultaneously inhibited by natural compounds $(27,28)$. One possibility for the differential regulation of IL- 6 and TNF- $\alpha$ by MEC is that IL- 6 and TNF- $\alpha$ possess a different promoter binding region for transcription factors. Previous studies have revealed that the signal transducer and activator of transcription (STAT) protein binding region is contained in the IL-6 promoter region, but not in the TNF- $\alpha$ promoter (29), and MEC may be a regulator of STAT signaling.

Several intracellular signaling pathways are associated with the increased expression of iNOS and pro-inflammatory cytokines $(2,20,30)$. In particular, MAPKs, including p38, ERK and JNK are important in the production of various inflammatory mediators $(7,30)$. LPS treatment of murine macrophages significantly enhances the production of inflammatory mediators via MAPK phosphorylation and stimulation of the downstream signaling pathway $(2,5,6)$. These studies imply that inhibition of p38, ERK and JNK phosphorylation may be a potential target pathway for the alleviation of severe inflammatory states. However, several studies have suggested that MAPK signaling cascades may be differently involved in the response of anti-inflammatory compounds in macrophages (31-33). In particular, a study by Watters et al demonstrated that the MEK/ERK pathway is not essential for the production of iNOS and IL-1 $\beta$ in macrophages (34). In the present study, MEC inhibited the LPS-induced phosphorylation of p38 and JNK, but not ERK, in a dose-dependent manner. However, the total MAPK levels were unchanged. Collectively, these results suggested that the activity of MAPKs, including p38 and JNK, rather than the expression of MAPKs, is the key regulatory mechanism underlying the MEC-mediated inhibition of inflammatory mediators.

$\mathrm{NF}-\kappa \mathrm{B}$ is the other major regulatory signaling molecule for inflammation. Following LPS stimulation, which leads to the phosphorylation and degradation of I $\kappa \mathrm{B}$ in the cytosol, NF- $\kappa \mathrm{B}$ subunits are freely translocated into the nucleus $(35,36)$. The nuclear translocated NF- $\mathrm{NB}$ subunits, p65 and p50, regulate the production of various inflammatory mediators, including TNF- $\alpha$, IL-6 and NO $(37,38)$. In the present study, stimulation of macrophages by LPS led to the activation of $N F-\kappa B$ and MAPKs. However, MEC had no effect on the activity of $\mathrm{NF}-\kappa \mathrm{B}$ or on the degradation of I $\mathrm{B}$ in the LPS-stimulated RAW 264.7 macrophages. NF- $\kappa \mathrm{B}$ and MAPK signaling share TLR4 adaptor molecules and accessory molecules, including myeloid differentiation primary response 88 (MyD88), toll/IL-1 receptor domain-containing adapter-inducing interferon- $\beta$ (TRIF), tumor-necrosis factor receptor-associated factor 6 (TRAF6)and IL receptor-associated kinase 1. However, the 
downstream signaling molecules of MyD88, TRIF and TRAF6 are quite different in the NF- $\mathrm{KB}$ and MAPK signaling pathways, therefore, the present study hypothesized that MEC may selectively regulate JNK and p38, but not the TLR4 accessory molecules.

The present study examined the regulation of inflammatory mediators by MEC in activated macrophages. Since macrophages are important in the pathogenesis of numerous inflammatory diseases, the MEC-mediated selective regulation of inflammatory mediators suggests that MEC may have therapeutic potential against inflammatory diseases. However, further studies are required to analyze the major components that are responsible for the reduction of inflammatory mediators and to elucidate the exact mechanism underlying the difference in the production of pro-inflammatory cytokines, IL- 6 and TNF- $\alpha$.

\section{Acknowledgements}

This study was supported by the National Research Foundation of Korea grant funded by the Korean government (Ministry of Education, Science and Technology; grant nos. NRF-2012R1A2A2A01047338 and NRF-2013R1A1A2062389).

\section{References}

1. Fujiwara $\mathrm{N}$ and Kobayashi $\mathrm{K}$ : Macrophages in inflammation. Curr Drug Targets Inflamm Allergy 4: 281-286, 2005.

2. Schroder K, Sweet MJ and Hume DA: Signal integration between IFNgamma and TLR signalling pathways in macrophages. Immunobiology 211: 511-524, 2006.

3. Aderem A and Underhill DM: Mechanisms of phagocytosis in macrophages. Annu Rev Immunol 17: 593-623, 1999.

4. Aderem A and Ulevitch RJ: Toll-like receptors in the induction of the innate immune response. Nature 406: 782-787, 2000.

5. Cario E, Rosenberg IM, Brandwein SL, Beck PL, Reinecker HC and Podolsky DK: Lipopolysaccharide activates distinct signaling pathways in intestinal epithelial cell lines expressing Toll-like receptors. J Immunol 164: 966-972, 2000.

6. Fujihara M, Muroi M, Tanamoto K, Suzuki T, Azuma H and Ikeda H: Molecular mechanisms of macrophage activation and deactivation by lipopolysaccharide: roles of the receptor complex. Pharmacol Ther 100: 171-194, 2003.

7. Hanada T and Yoshimura A: Regulation of cytokine signaling and inflammation. Cytokine Growth Factor Rev 13: 413-421, 2002.

8. Nathan C and Xie QW: Regulation of biosynthesis of nitric oxide. J Biol Chem 269: 13725-13728, 1994.

9. MacMicking J, Xie QW and Nathan C: Nitric oxide and macrophage function. Annu Rev Immunol 15: 323-350, 1997.

10. Clancy RM, Amin AR and Abramson SB: The role of nitric oxide in inflammation and immunity. Arthritis Rheum 41: 1141-1151, 1998.

11. Moncada S: Nitric oxide: discovery and impact on clinical medicine. J R Soc Med 92: 164-169, 1999.

12. Dinarello CA: Anti-inflammatory agents: present and future. Cell 140: 935-950, 2010.

13. Eke T, Al-Husainy $\mathrm{S}$ and Raynor MK: The spectrum of ocular inflammation caused by Euphorbia plant sap. Arch Ophthalmol 18: 13-16, 2000.

14. Lazarini CA, Uema AH, Brandão GM, Guimarães AP and Bernardi MM: Croton zehntneri essential oil: effects on behavioral models related to depression and anxiety. Phytomedicine 7: 477-481, 2000

15. Thyagarajan SP, Subramanian S, Thirunalasundari T, Venkateswaran PS and Blumberg BS: Effect of Phyllanthus amarus on chronic carriers of hepatitis B virus. Lancet 1: 764-766, 1988.

16. Suarez AI, Compagnone RS, Salazar-Bookaman MM, et al: Antinociceptive and anti-inflammatory effects of Croton malambo bark aqueous extract. J Ethnopharmacol 88: 11-14, 2003.
17. Pereira U, Garcia-Le Gal C, Le Gal G, et al: Effects of sangre de drago in an in vitro model of cutaneous neurogenic inflammation. Exp Dermatol 19: 796-799, 2010.

18. Risco E, Ghia F, Vila R, Iglesias J, Alvarez E and Cañigueral S: Immunomodulatory activity and chemical characterisation of sangre de drago (dragon's blood) from Croton lechleri. Planta Med 69: 785-794, 2003.

19. Nathan C and Xie QW: Nitric oxide synthases: roles, tolls, and controls. Cell 78: 915-918, 1994.

20. Szabo $C$ and Thiemermann C: Regulation of the expression of the inducible isoform of nitric oxide synthase. Adv Pharmacol 34: 113-153, 1995.

21. Bosca L, Zeini M, Traves PG and Hortelano S: Nitric oxide and cell viability in inflammatory cells: a role for NO in macrophage function and fate. Toxicology 208: 249-258, 2005.

22. Kröncke KD, Fehsel K and Kolb-Bachofen V: Inducible nitric oxide synthase in human diseases. Clin Exp Immunol 113: 147-156, 1998.

23. Guadagni F, Ferroni P, Palmirotta R, Portarena I, Formica V and Roselli M: Review. TNF/VEGF cross-talk in chronic inflammation-related cancer initiation and progression: an early target in anticancer therapeutic strategy. In Vivo 21: 147-161, 2007.

24. Nishimoto $\mathrm{N}$ and Kishimoto $\mathrm{T}$ : Interleukin 6: from bench to bedside. Nat Clin Pract Rheumatol 2: 619-626, 2006.

25. Kavanaugh A: Interleukin-6 inhibition and clinical efficacy in rheumatoid arthritis treatment - data from randomized clinical trials. Bull NYU Hosp Jt Dis 65: S16-S20, 2007.

26. Straub RH, Harle P, Yamana S, et al: Anti-interleukin-6 receptor antibody therapy favors adrenal androgen secretion in patients with rheumatoid arthritis: a randomized, double-blind, placebo-controlled study. Arthritis Rheum 54: 1778-1785, 2006.

27. Samavati L, Rastogi R, Du W, Huttemann M, Fite A and Franchi L: STAT3 tyrosine phosphorylation is critical for interleukin 1 beta and interleukin- 6 production in response to lipopolysaccharide and live bacteria. Mol Immunol 46: 1867-1877, 2009.

28. Zhu ZG, Jin H, Yu PJ, Tian YX, Zhang JJ and Wu SG: Mollugin inhibits the inflammatory response in lipopolysaccharide-stimulated RAW264.7 macrophages by blocking the janus kinase-signal transducers and activators of transcription signaling pathway. Biol Pharm Bull 36: 399-406, 2013.

29. Lee C, Lim HK, Sakong J, Lee YS, Kim JR and Baek SH: Janus kinase-signal transducer and activator of transcription mediates phosphatidic acid-induced interleukin (IL)-1beta and IL-6 production. Mol Pharmacol 69: 1041-1047, 2006.

30. Stalinska K, Guzdek A, Rokicki M and Koj A: Transcription factors as targets of the anti-inflammatory treatment. A cell culture study with extracts from some Mediterranean diet plants. J Physiol Pharmaco 56: S157-S169, 2005.

31. Burk DR, Senechal-Willis P, Lopez LC, Hogue BG and Daskalova SM: Suppression of lipopolysaccharide-induced inflammatory responses in RAW 264.7 murine macrophages by aqueous extract of Clinopodium vulgare L. (Lamiaceae). J Ethnopharmacol 126: 397-405, 2009.

32. Liu H, Bargouti M, Zughaier S, et al: Osteoinductive LIM mineralization protein-1 suppresses activation of NF-kappaB and selectively regulates MAPK pathways in pre-osteoclasts. Bone 46: 1328-1335, 2010.

33. Shan J, Fu J, Zhao Z, et al: Chlorogenic acid inhibits lipopolysaccharide-induced cyclooxygenase-2 expression in RAW264.7 cells through suppressing NF-kappaB and JNK/AP-1 activation. Int Immunopharmacol 9: 1042-1048, 2009.

34. Watters JJ, Sommer JA, Pfeiffer ZA, Prabhu U, Guerra AN and Bertics PJ: A differential role for the mitogen-activated protein kinases in lipopolysaccharide signaling: the MEK/ERK pathway is not essential for nitric oxide and interleukin lbeta production. J Biol Chem 277: 9077-9087, 2002.

35. Karin $\mathrm{M}$ and Ben-Neriah Y: Phosphorylation meets ubiquitination: the control of NF-[kappa]B activity. Annu Rev Immunol 18: 621-663, 2000.

36. Karin M and Delhase M: The I kappa B kinase (IKK) and NF-kappa B: key elements of proinflammatory signalling. Semin Immunol 12: 85-98, 2000.

37. Surh YJ, Chun KS, Cha HH, et al: Molecular mechanisms underlying chemopreventive activities of anti-inflammatory phytochemicals: down-regulation of COX-2 and iNOS through suppression of NF-kappa B activation. Mutat Res 480-481: 243-268, 2001.

38. Verma IM, Stevenson JK, Schwarz EM, Van Antwerp D and Miyamoto S: Rel/NF-kappa B/I kappa B family: intimate tales of association and dissociation. Genes Dev 9: 2723-2735, 1995. 